\title{
Asymmetric rolling of interstitial free steel sheets: microstructural evolution and mechanical properties
}

\author{
Saeed Tamimi ${ }^{\text {a,b }}$, Jose J. Gracio ${ }^{\mathrm{b}}$, Augusto B. Lopes ${ }^{\mathrm{c}}$, Said Ahzi ${ }^{\mathrm{b}, \mathrm{d}}$, and Frederic Barlat \\ ${ }^{a}$ Advanced Forming Research Centre (AFRC), University of Strathclyde, United Kingdom \\ ${ }^{\mathrm{b}}$ Centre for Mechanical Technology and Automation, Department of Mechanical Engineering, University of Aveiro, Portugal \\ ${ }^{\mathrm{c}}$ Departamento de Engenharia de Materiais e Cerâmica, CICECO, Universidade de Aveiro, Portugal \\ ${ }^{\mathrm{d}}$ ICUBE Laboratory/CNRS, University of Strasbourg, France \\ ${ }^{\mathrm{e}}$ Graduate Institute of Ferrous Technology, Pohang University of Science and Technology, Republic of Korea
}

\begin{abstract}
:
Asymmetric rolling (ASR) is a promising method for introducing shear deformation throughout the thickness of sheets. The induced shear deformation in ASR will result in texture evolution and which could also affect microstructural features controlling mechanical properties (such as the tensile strength). The main objective of this work consists of the investigation of the influence of ASR process on microstructure and texture evolution and their induced mechanical properties in interstitial free (IF) steel sheets. Both reverse and continuous asymmetric rolling were carried out to deform IF steel sheets. The results of optical microscopy observations showed no significant differences between the grain morphology of asymmetric and conventionally rolled (CR) samples. However, the obtained results of transmission electron microscopy (TEM) and electron backscatter diffraction (EBSD) indicated that fine and equiaxed microstructure was formed through the asymmetric rolling process. Texture evolution through plastic deformation has also been analyzed using X-ray diffraction. In addition, polycrystalline plasticity simulations were used to predict the texture evolution and the induced mechanical properties. The effect of the induced amount of pre-straining on the mechanical response of the samples through uniaxial tensile test has been studied. Results showed that at low thickness reductions (18\%) the asymmetric rolled sample presents higher stress than that of the conventionally rolled sheet; while for higher thickness reductions $(60 \%)$ the trend is reversed.
\end{abstract}

Keywords: asymmetric rolling; interstitial free steel; microstructure; crystallographic texture; mechanical properties; polycrystalline simulations

\section{Introduction}

Improving the mechanical properties is desirable for reducing the weight of engineering materials used in components. This can directly translate into significant economic gains. Such a challenge can be partially met

\footnotetext{
* Corresponding author: (Saeed Tamimi) Tel. +44(0)145345571 E-mail address: saeed.tamimi@strath.ac.uk

Advanced Forming Research Centre (AFRC), University of Strathclyde, United Kingdom
}

This is a peer-reviewed accepted author manuscript of the following research output: Tamimi, S., Gracio, J. J., Lopes, A. B., Ahzi, S., \& Barlat, F. (2018). Asymmetric rolling of interstitial free steel sheets: microstructural evolution and mechanical properties. Journal of Manufacturing Processes, 31, 583-592. [31]. DOI: 10.1016/j.jmapro.2017.12.014 
by reducing the grain size and controlling texture evolution. This has been has demonstrated by experiments that were performed with equal channel angular pressing [1], accumulative roll bonding [2] and high pressure torsion [3]. These previous works showed that shear plastic deformation applied through the methods, has a key role in grain refinement that allows for the production of the preferred texture in metallic materials. However, a weakness of these processes is the fact that they are conducted on samples with limited dimensions. This is a key factor that greatly affects their potential for industrial applications.

Recently, the Asymmetric rolling (ASR) process has been introduced. This process is able to produce ultrafine grain materials as well as develop desirable crystallographic texture with enhanced performance in metallic sheets for particular applications [4]. The principal base of ASR is the elimination of symmetries of conventional rolling $(\mathrm{CR})$ process which causes shear deformation throughout the thickness of the metallic sheet. Kim and Lee indicated that various configurations can be used including different circumferential velocities of the two working rolls due to their different diameters or rotation speeds [5]. Also, Utsunomiya et al. introduced shear plastic deformation throughout the thickness of metallic sheets by applying different lubrication conditions [6]. In our previous study, the effect of ASR and heat treatment on texture modification of an aluminium alloy and subsequently on their mechanical response has been taken into consideration [7]. Furthermore, it was indicated that optimizing the process parameters, e.g. thickness reduction per pass, velocity ratios and contact conditions between the rolls and sample surface, can increase the shear plastic deformation through the sheet thickness [8]. The shear plastic deformation produced the desired texture, which resulted in enhanced mechanical properties in aluminium-magnesium alloy sheets. There have been a number of publications in recent years on the ASR process being used on steel sheets. Lee and Lee [9] employed the ASR at high temperatures on IF steel sheets and observed the impact of extra shear strain on texture evolution of IF steel during warm-ASR process whereas in the work of Wauthier et al. [10], the influence of ASR on the texture evolution during post annealing of recrystallization in the same material has been taken into account. In addition, Ding et al. used ASR process to produce an ultrafine structure in commercially available iron sheets, with the goal of increasing the strength of metallic sheets [11]. Cai et al. took the advantage of ASR and dynamic transformation to produce a gradient-distributed ultrafine grain ferrite and martensite duplex structure in micro-alloyed steel sheets [12]. In spite of previous studies regarding the ASR of steels, the impact of shear plastic deformation introduced in ASR and the effect of strain path changing in multi pass ASR process on microstructure of steels and their induced mechanical properties have not been systematically investigated. In the present study, IF steel sheets were asymmetrically rolled and the influence of shear deformation and strain path changing on the micro- and macro- properties of the specimens is investigated. 


\section{Experimental procedures and modelling}

The material used in the present study was $1.2 \mathrm{~mm}$ thick IF steel sheet. The annealed sheet had an average grain size of about $25 \mu \mathrm{m}$. The chemical composition is given in Table 1.

Table 1. Chemical composition of as received IF steel.

\begin{tabular}{|c|c|c|c|c|c|c|}
\hline $\mathbf{C}$ & $\mathbf{T i}$ & $\mathbf{V}$ & $\mathbf{N i}$ & $\mathbf{M n}$ & $\mathbf{C o}$ & $\mathbf{M o}$ \\
\hline $0.004 \%$ & $0.040 \%$ & $0.001 \%$ & $0.017 \%$ & $0.061 \%$ & $0.002 \%$ & $0.002 \%$ \\
\hline
\end{tabular}

The as-received samples were subjected to four ASR (and CR) passes leading to $60 \%$ of total reduction. The ASR process was carried out using a velocity ratio equal to 1.5 of upper to lower working-rolls ( 4 and 6 rotates per minutes as absolute speeds for the working-rolls) and without lubrication. We designated these plastic deformation routes as CR for conventional rolling and two types of ASR:

- Reverse asymmetric rolling (R-ASR) in which the rolling direction was reversed after each step.

- Continuous asymmetric rolling (C-ASR) in which the samples were not reversed with respect to rolling direction.

Schematics of these processes are shown in Figure 1.

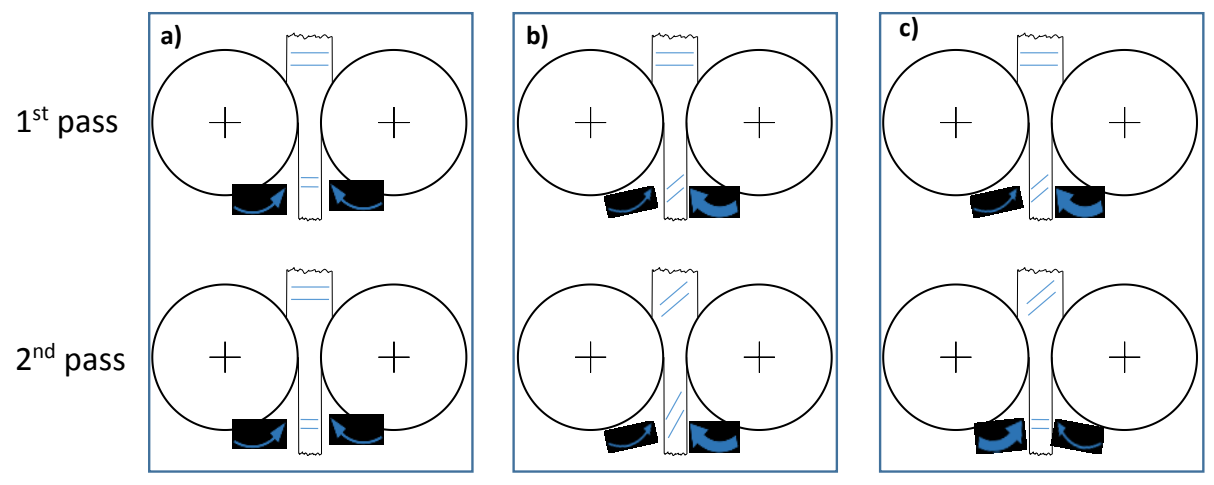

Figure 1. Schematics of two passes of: a) $C R, b) C$-ASR and c) R-ASR processes

The resulting microstructure was investigated by optical microscopy and TEM. In addition, crystallographic texture measurement was performed using an X-ray diffractometer; with an X-ray texture goniometer, at the mid-thickness locations of the sheets. With the aim of studying the effect of using different strain paths on the microstructure and mechanical behaviors, heat treatment of recovery have also been carried out. For that end, the rolled samples were placed in an electrical furnace at $550^{\circ} \mathrm{C}$ for 60 minutes. Subsequently, the influence of the heat treatment on the microstructure evolution was studied using EBSD analyses. Furthermore, tensile test specimens were prepared along the rolling direction (RD) from CR and C-ASR sheets and the tensile tests were conducted at ambient room condition and an engineering strain rate of $10^{-4} \mathrm{~s}^{-1}$ was used. In the last round of experiments, the influence of the total reduction of rolling (i.e. CR vs. C-ASR) on 
their mechanical behavior was investigated by imposing total thickness reductions of $18 \%, 36 \%$ and $60 \%$ followed by a recovery annealing as described previously.

The viscoplastic self-consistent (VPSC) model was employed in order to study the texture evolution through different types of rolling as described before. The VPSC model was firstly proposed by Molinari et al. [13] and was later developed by Lebensohn and Tome [14]. In this modeling, plasticity of the BCC single crystals of IF steel occurs by crystallographic slip on $\{110\}<111>,\{112\}<111>,\{123\}<111>$ slip systems. The initial critical resolved shear stress is assumed the same for all slip systems.

The initial sample texture that was obtained from the X-ray data were used as input files for the VPSC code. The macroscopically imposed velocity gradient for the sheets under CR and ASR processes is approximated by plane strain compression for CR and combined plane strain compression and shear for ASR (Equation 1):

$$
L_{C R}=\left[\begin{array}{ccc}
\dot{e}_{11} & 0 & 0 \\
0 & 0 & 0 \\
0 & 0 & \dot{e}_{33}
\end{array}\right], \quad L_{A S R}=\left[\begin{array}{ccc}
\dot{e}_{11} & 0 & \dot{e}_{13} \\
0 & 0 & 0 \\
0 & 0 & \dot{e}_{33}
\end{array}\right]
$$

Equation 1

Here, $\dot{e}_{11}, \dot{e}_{33}$ and $\frac{1}{2} \dot{e}_{13}$ are strain rate components with indices 1, 2 and 3 representing the normal, transvers and rolling directions, respectively. The tensor $L$ presents the macroscopic velocity gradient. The through thickness shear strain under each pass of ASR was measured. To this end, a number of lines were scratched on the side (TD plane) of the sheet samples perpendicular to the ND plane using a metallic pen. During the ASR process, the lines become inclined by an angle with respect to the ND axis due to the shear plastic deformation as shown in Figure 2. The measured inclination of the lines represents the additional shear deformation induced by ASR allowing us to evaluate the shear component of the velocity gradient tensor of ASR process.

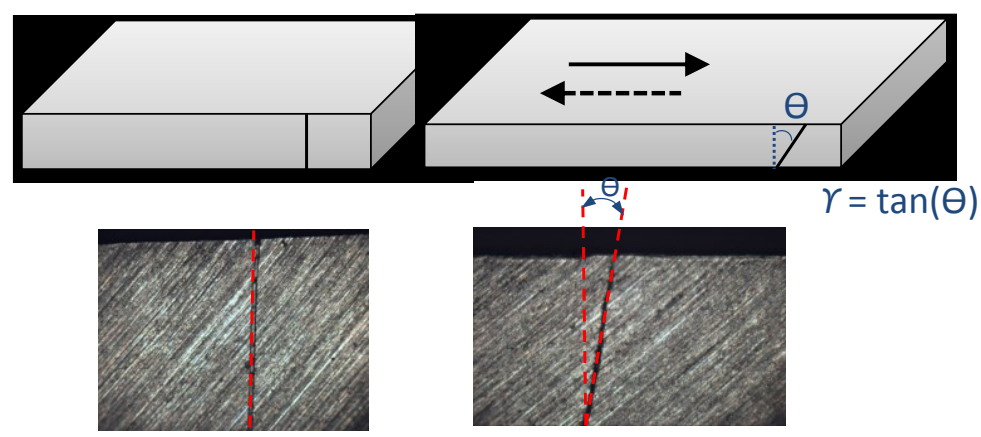

Figure 2. Inclined line toward shear induced by ASR

Furthermore, the VPSC model was used to simulate the tensile stress-strain curves of different samples and the predicted results were compared to the experimental ones. For that, the threshold resolved shear stressaccumulated shear strain relationship of a single crystal $(\tau-\Gamma)$ proposed by Tome et al. [15] was used: 


$$
\tau=\tau_{0}+\left(\tau_{1}+\theta_{1} \Gamma\right)\left(1-\exp \left(-\Gamma\left|\frac{\theta_{0}}{\tau_{1}}\right|\right)\right) \quad \text { Equation } 2
$$

Here, $\tau_{0}, \theta_{0}, \theta_{1}$ and $\left(\tau_{0}+\tau_{1}\right)$ are the initial critical resolved shear stress (CRSS), the initial strain hardening rate, the asymptotic hardening rate and the back-extrapolated CRSS, respectively. To determine these coefficients, we used tensile stress-strain curve of the CR sample as a reference and the values are therefore obtained by fitting, as presented in Table 2 .

Table 2. Material constants for the IF steel sample in Equation 2.

\begin{tabular}{|c|c|c|c|}
\hline$\tau_{0}$ & $\theta_{0}$ & $\theta_{1}$ & $\tau_{1}$ \\
\hline $187 \mathrm{MPa}$ & $985 \mathrm{MPa}$ & $17 \mathrm{MPa}$ & $43 \mathrm{MPa}$ \\
\hline
\end{tabular}

\section{Results and discussion}

\subsection{Texture development under plastic deformation}

In this section, the crystallographic textures are shown by ODFs in the Euler space represented by $\mathrm{Phi}_{1}$, $\mathrm{Phi}$ and $\mathrm{Phi}_{2}$ angles (Bunge's notation) [16]. Usually, the Euler space section at $\mathrm{Phi}_{2}=45^{\circ}$ is only presented since the most important texture components of $\mathrm{BCC}$ materials are in this section. Figure 3 shows the $\mathrm{Phi}_{2}=45^{\circ}$ section of the ODF presentation of the sheets deformed using CR and C-ASR. In order to facilitate the interpretation of the developed texture components in the study, the ideal components in $\mathrm{ODF} \mathrm{Phi}_{2}=45^{\circ}$ section were displayed in the ODF presentations. The simulated texture of CR is shown in Figure 3a. The strong gamma fiber can clearly be seen in which $E\{111\}<110>$ components are more pronounced (Figure 3a). The alpha fiber including the i $\{112\}<-110>$ component can also be detected. Note that this type of texture (i.e. rolling texture) has also been reported by others; e.g. the investigation of Holscher et al. [17]. However, the crystallographic texture of the C-ASR sample is more uniform and relatively weaker in comparison to the intense texture components of the CR sample (see Figure $3 a$ and $b$ ). The $F\{111\}<11-2>$ and the weak i $\{112\}<-110>$ can be detected in this ODF section. The shear strain imposed through C-ASR has caused the texture components to deviate from the ideal texture components. The measured textures from both processes are shown in Figure $3 c$ and $d$. The simulated texture of $C R$ is qualitatively in agreement with the measured texture. However, discrepancy between the predicted and the experimental textures is obtained for the sample subjected to C-ASR. 


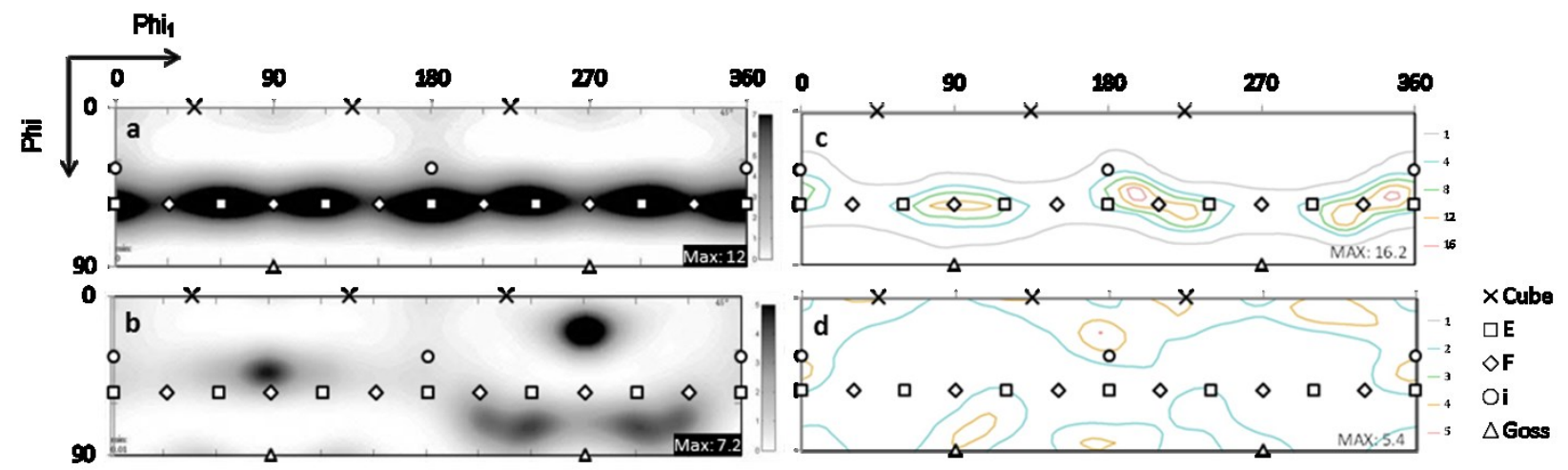

Figure 3. Crystallographic texture ( $P h i_{2}=45^{\circ}$ section) simulated by VPSC model: (a) CR, (b) C-ASR; and measured from experiments of (c) $C R$ and (d) C-ASR.

\subsection{Microstructure evolution}

Figure 4 illustrates the microstructure of C-ASR and CR samples after $60 \%$ reduction taken with an optical microscope. After both C-ASR and CR the grains appear elongated in the rolling direction. According to these results, no significant difference between the grains' size and shape in the investigated samples can be detected. However, in Figure 4a the darker regions appear with a wrinkled skin covering the elongated grains in the C-ASR sample. The observed feature may be associated with a different dislocation organization of the C-ASR sample, which could be due to the shear strain. The shear strain imposed through C-ASR causes the activation of different slip systems than those activated through $\mathrm{CR}$, which result in different dislocation organizations. In order to study the dislocation organization of the metallic sheets deformed by C-ASR and CR, a TEM observation was carried out.
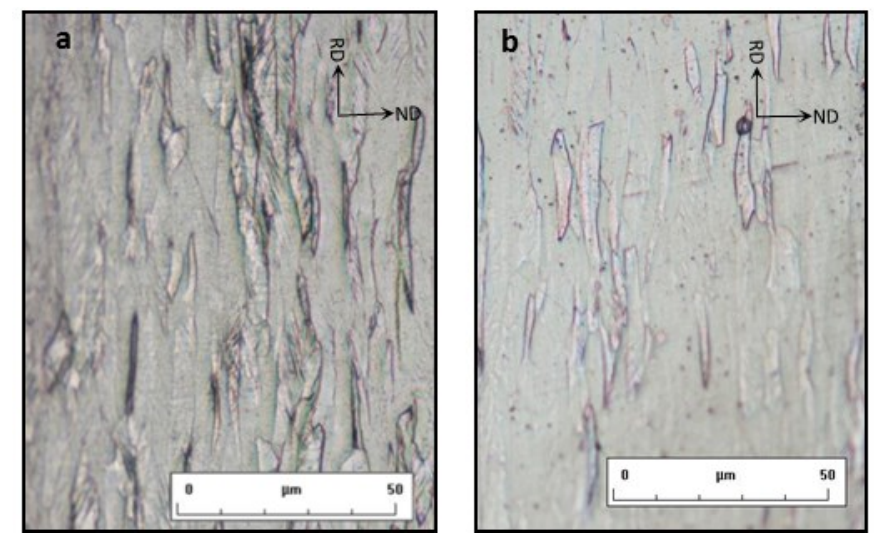

Figure 4. Optical microscopy observation of $60 \%$ rolled samples of a) C-ASR and b) CR specimens.

The TEM observation of the 60\%-reduction rolled samples using C-ASR and CR at different points are shown in Figure 5a and b, respectively. The substructure of C-ASR sample in Figure 5a is characterized by dislocation walls which form equiaxed dislocation cells, whereas the dislocation cells of CR sample 
are elongated along the RD (Figure 5b). In addition, the dislocation cells produced during C-ASR are relatively smaller than those produced during CR. This is in agreement with the observation of Ding et al. [11]. The shear strain involved during C-ASR process allows for the formation of fine and equiaxed cells. For the same amount of reduction in sheet thickness (i.e. 60\%), C-ASR introduces more equivalent strain than CR. This is due to additional shear plastic deformation being induced. Optimizing the ASR process to achieve more shear plastic deformation results in the introduction of more severe plastic deformation (i.e. SPD) [11]. It is for this reason that fine and equiaxed microstructure in C-ASR can be achieved by CASR. At the initial stage, a tangle of dislocations is formed inside the coarse grains which then provides the geometrically necessary boundaries are arranged to subdivide the grains into the dislocation cells. The new dislocation cell boundaries originate from accumulation and rearrangement of dislocations during plastic deformations [18] [19] [20] [21]. Cui and Ohori showed that the shear plastic deformation in ASR is more effective in the formation of fine and equiaxed grains than equivalent strain [22]. 
a)

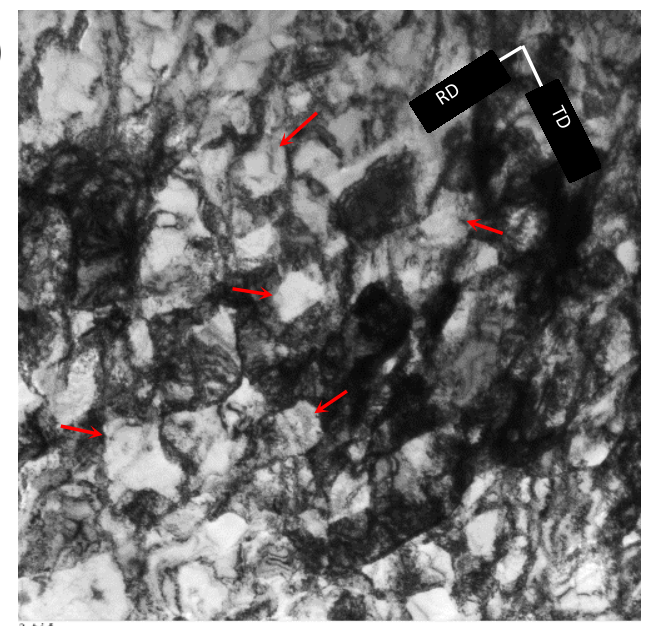
3.tif
Print Mag: $23600 \times 07.0$ in
Trm Mode: Imaging

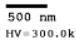

HV $3000.0 \mathrm{kV}$
Direct hag: $3000 \mathrm{x}$

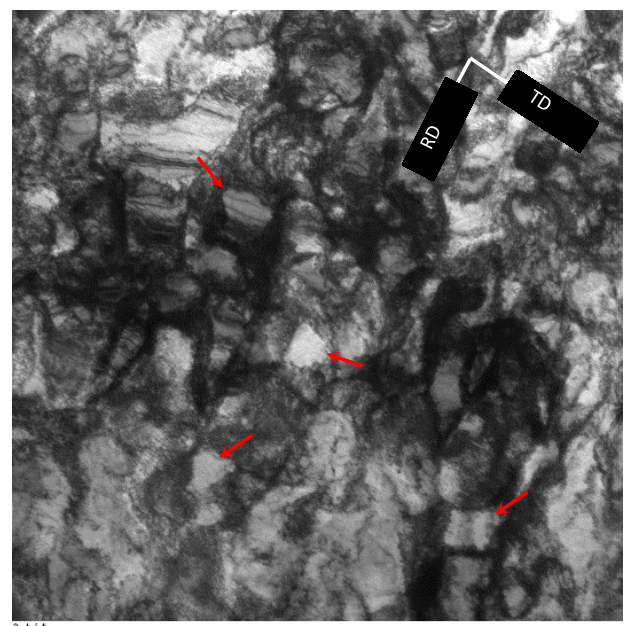

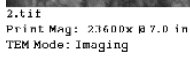

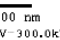

Dirve: Mag: anox
university of \&veiro

b)
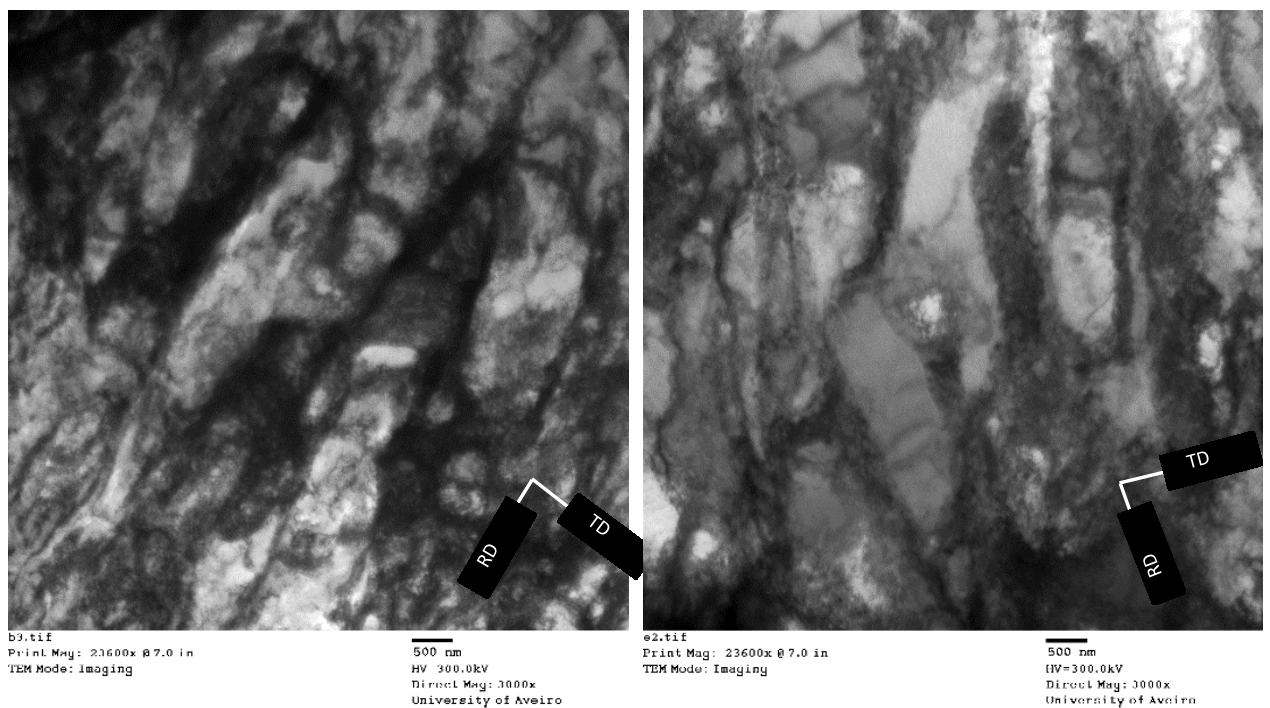

Figure 5. TEM observation of the sample rolled for $60 \%$ reduction with: a) C-ASR and b) and CR.

It should be noted that in some parts of the surface regions of the CR sheet sample, the equiaxed substructure can also be detected. This is associated with the shear deformation imposed by friction between sheet and the working-roll surfaces. However, due to the fact that only small parts of the CR sample sheet thickness experienced the shear deformation induced by friction, the majority of the sheet thickness is characterized by elongated substructure as shown in Figure $5 b$.

Figure 6 illustrates the influence of reversing the sample between the ASR passes (i.e. R-ASR process) on the TEM microstructure of the sample. A significant number of dislocations cover the observed area which are not well-organized. However, there are some regions in this micrograph that are showing newly formed dislocation walls. These rearranged the dislocations and form imprecise equiaxed cells. The new dislocation 
cells are larger in comparison with those observed in the C-ASR sample (see Figure 5a and Figure 6). At first sight, these results seem to be in contradiction with the previous studies reported by Iwahashi et al. in that study it was reported that changes in strain path should promote the fine substructure [23] [24]. However, this approach is not generally accepted. In fact, Gholinia et al. has shown that a constant strain path is the most effective way to form fine grained structures for metallic sheets [25].

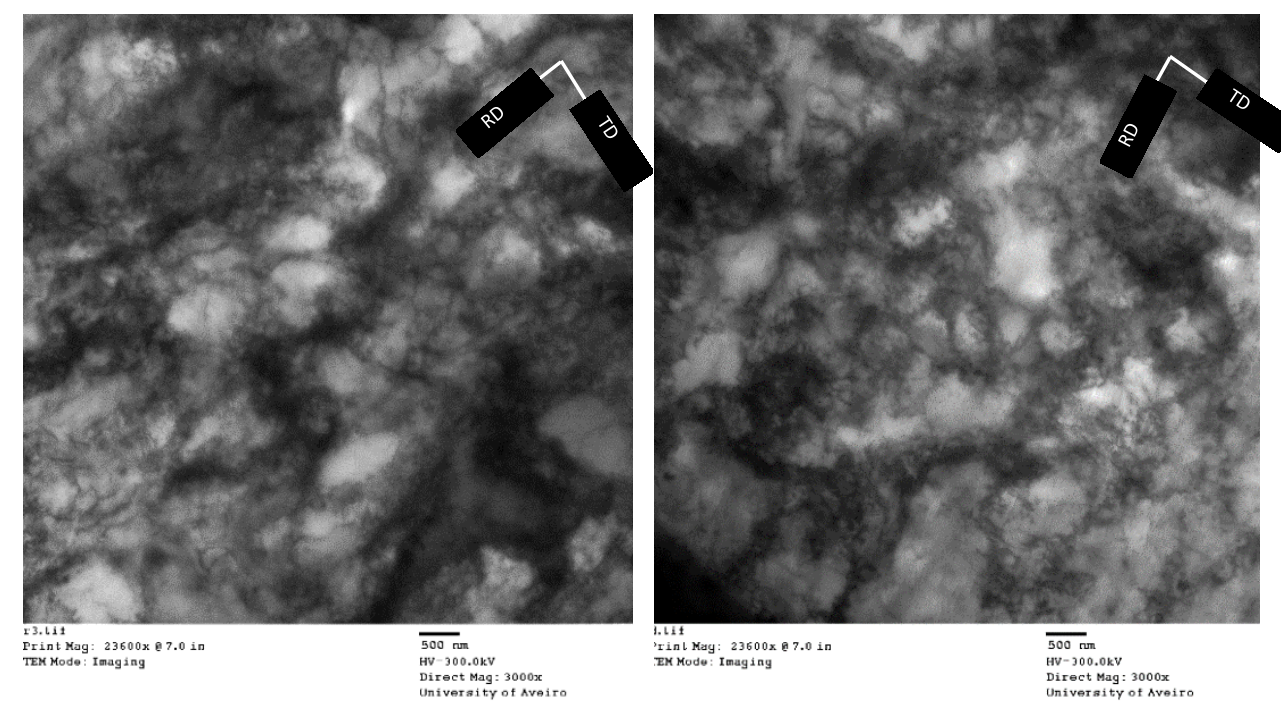

Figure 6. TEM observation of the sample rolled for $60 \%$ reduction with R-ASR process.

Figure 5 and Figure 6 presented the TEM substructure that was induced by various plastic deformation processes where the important characteristic feature is the various strain routes. In order to study the effect of strain path change on the activity of slip systems in each process, we analyse the so-called $\alpha$ parameter of CR, R-ASR and C-ASR (as defined by Schmitt et al. [26]). This parameter represents the cosine of the angle between the strain routes of two successive passes. It also provides an estimate of the impact of strain path change on the activity of different slip systems (see details in Appendix). Regarding the calculated $\alpha$ parameters for each process - as shown in the Appendix $\left(\alpha_{C R}=\alpha_{C-A S R}=1\right.$ and $\left.\alpha_{R-A S R}<1\right)$, it can be inferred that slip system activities of the CR and the C-ASR processes do not change between the passes; i.e. slip occurs on the same crystallographic plane and direction in all passes. Nevertheless, changing the sample direction between R-ASR passes leads to the activation of new slip systems and previous slip systems movement in opposite directions. This may result in a substructure in R-ASR sheets which is not well organized (Figure 6) compared to that in C-ASR (Figure 5a). 
In order to avoid early sample fracture during the tensile test, therefore, allowing for the observation of the resulting microstructure and texture on the mechanical behaviour, a recovery heat-treatment was applied on the pre-deformed samples. The annealing temperature should be high enough to annihilate dislocation from the dislocation cells but should not be higher than recrystallization temperature. Working within these parameters will allow for the developed texture during plastic deformation to be retained. It was experimentally observed that annealing at $550^{\circ} \mathrm{C}$ for 60 minutes is an appropriate condition for recovery heat treatment for these rolled samples. The EBSD analysis of the C-ASR sample that were recovery heat treated shown in Figure 7, indicates small and equiaxed grains. These grains are free of dislocations and possess high angle boundaries. It can be seen that those dislocations which have been produced through plastic deformation and distributed inside dislocation cells, were annihilated and reorganized in the boundaries during recovery heat-treatment. Since small and equiaxed dislocation cells formed through C-ASR (as seen in Figure 5a), the grains and sub-grains after the heat treatment are small and have equiaxed shapes.
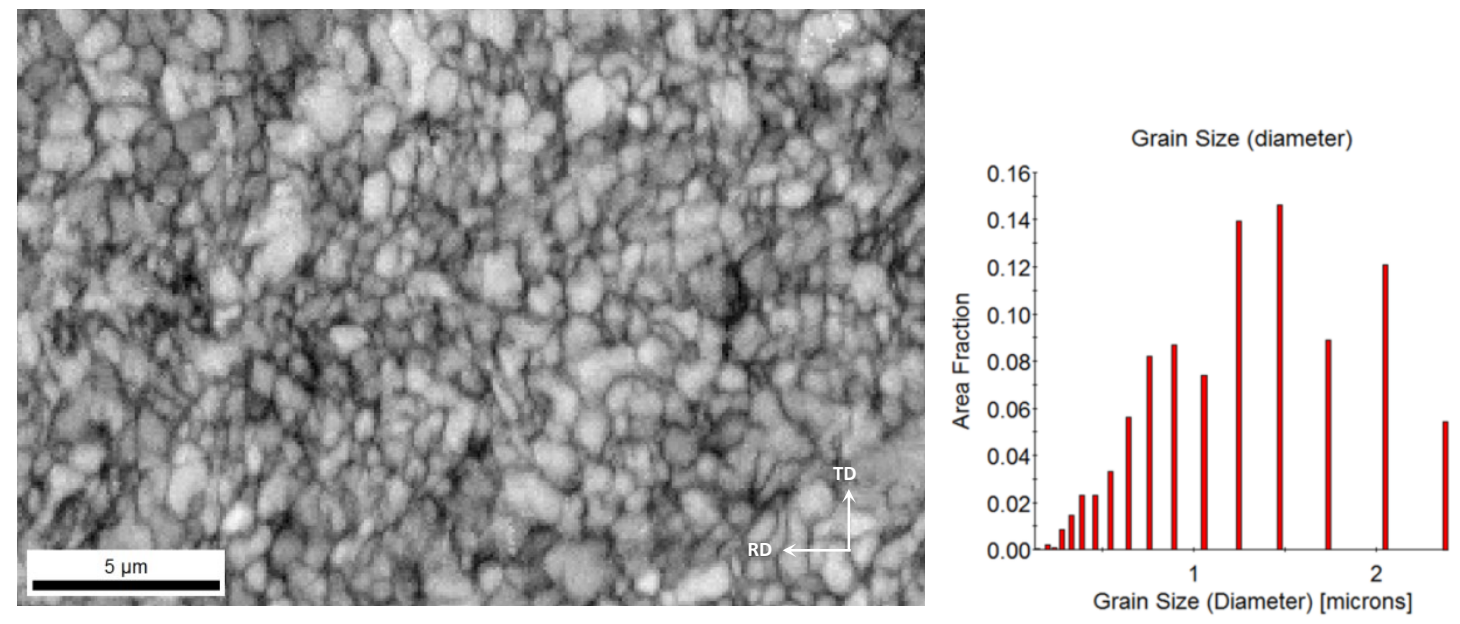

Figure 7. EBSD observation of the sample rolled for $60 \%$ reduction with C-ASR process.

\subsection{Total thickness reduction effects}

Figure 8 shows the stress-strain curves (tensile test along the RD) for the samples deformed under CR and C-ASR. It shows the different reductions per pass after annealing. Each sample was subjected to 4 passes giving rise to a total deformations ranging approximately from $18 \%$ to $60 \%$. It can be noticed that for low reduction (18\%), the stress-strain curve for the C-ASR sample presents higher stresses than the CR sample. However, for the $60 \%$ reduction, this is reversed and the higher stress levels were detected in the CR sheet. Such a phenomenon leads to the appearance of a transient behaviour for reduction 
ratios of around $36 \%$. Figure 8 shows that the elongation of the tensile samples is higher for the CR at low thickness reduction and is the same for both CR and C-ASR for the high reduction.

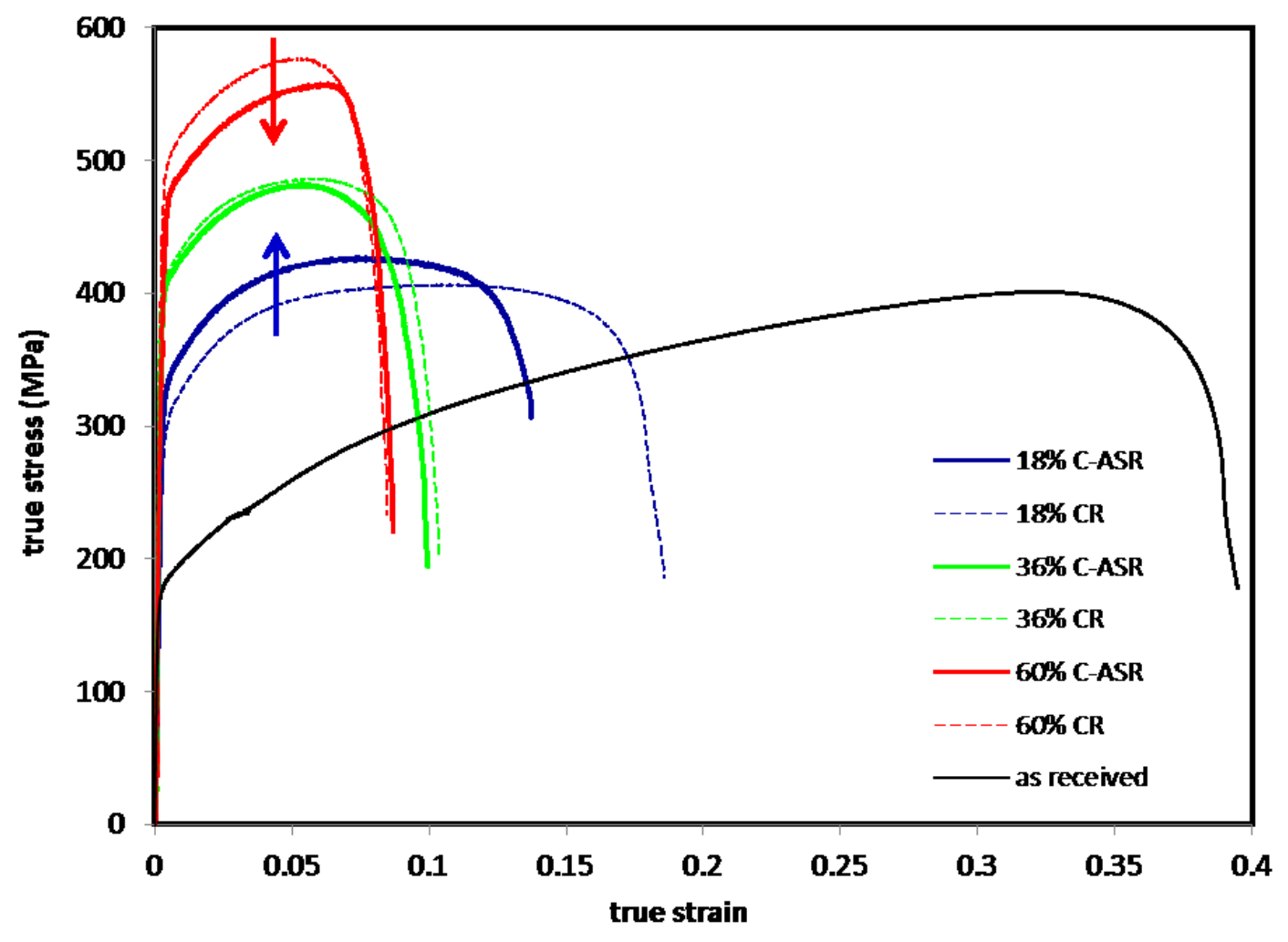

Figure 8. True stress- true strain curves of $C R$ and C-ASR samples after annealing along the RD.

Furthermore, the Lankford coefficients (R-value) of the deformed samples along the rolling direction (RD) are given in Table 1. The R-value is defined as the ratio of plastic strain in the width direction to that in the thickness direction of the sheet under tensile loading.

$$
R=\frac{\varepsilon_{w}}{\varepsilon_{t}} \quad \text { Equation } 3
$$

Here $\varepsilon_{w}$ and $\varepsilon_{t}$ are true plastic strain in the width and thickness of the tensile test sample, respectively. The results in Table 3 show that imposing shear strain in addition to compression strain may lead to a slight increase in the R-value of the deformed sheets for low and high sheet thickness reductions. However, at $36 \%$ reduction, the R-values of both samples are approximately the same.

Table 3. $R$-value of different reductions of $C R$ and $C$-ASR along the $R D$.

\begin{tabular}{|l|l|l|l|}
\hline $\begin{array}{c}\text { Total reduction } \\
\text { process }\end{array}$ & $18 \%$ & $36 \%$ & $60 \%$ \\
\hline
\end{tabular}




\begin{tabular}{|l|l|l|l|}
\hline C-ASR & 0.75 & 0.80 & 0.78 \\
\hline CR & 0.65 & 0.81 & 0.62 \\
\hline
\end{tabular}

As previously indicated, the microstructure and crystallographic texture are the factors that determine the mechanical properties. The recovery heat-treatment results in a rearrangement of the substructure induced by the plastic deformation processes (Figure 5). This causes misorientation angles of dislocation cell walls to increase and form a small and equiaxed morphology microstructure (Figure 7). Additionally, crystallographic orientations of the deformed sheets remained unchanged throughout the described recovery heat treatment. The texture of C-ASR and CR samples presented in Figure 3 indicated that the rolling texture (particularly $\mathrm{i}$, the gamma fiber), in the CR sample appeared with high intensities, while the C-ASR samples had weak texture. Consequently, the resulting features observed after low temperature annealing of the C-ASR samples in comparison with the CR ones, is a fine and equiaxed microstructure and a relatively less intense texture and deviated from rolling texture.

In order to evaluate the impact on the mechanical response of the produced texture, crystal plasticity simulations were performed using the VPSC model. For this purpose, the measured crystallographic textures from experiments of $60 \%$ reduction CR and C-ASR samples (Figure 3) were used for simulation through uniaxial tensile test along the rolling direction. The calculated true stress- true strain curves of the CR and C-ASR samples are shown in Figure 9. The calculated mechanical behaviour of the CR samples possesses higher stress than the C-ASR sample in each strain of tension. The experimental true stresstrue strain curve of the sample pre-deformed by $60 \%$ reduction of CR process is also shown in Figure 9 .

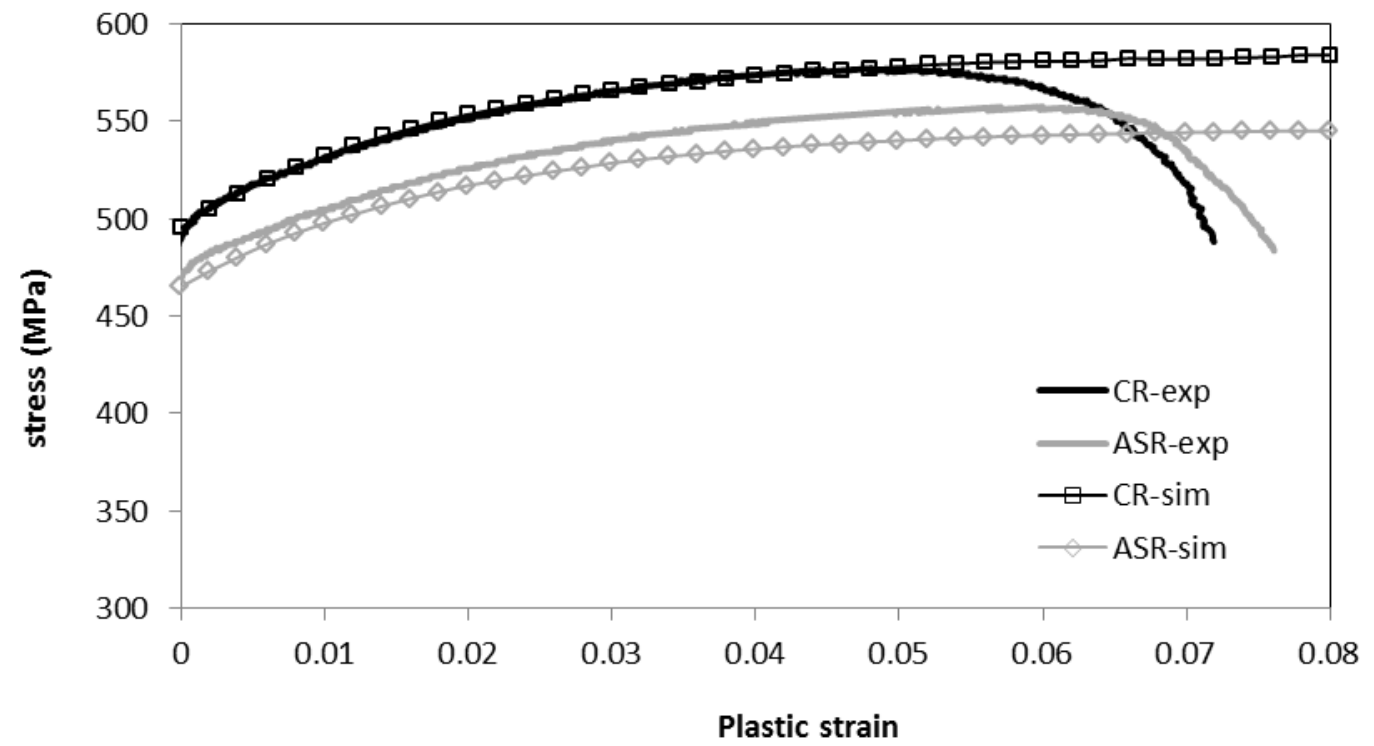

Figure 9. Simulated true stress-true strain curves obtained with VPSC model of CR and C-ASR samples along the RD. 
With the purpose of understanding the role of different texture components on the macroscopic behaviour, VPSC modelling was employed to simulate the behaviour of each individual component of $\mathrm{BCC}$ materials through a uniaxial tensile test in RD as shown in Figure 10. As shown, the $<M>$ of the $i$ and E texture components present a similar trend and are higher than other types of texture components in each particular strain. Hence in Figure 9, the higher stress of CR is attributed with the strong $i$ and $E$ components of CR sample relatively to the C-ASR sample.

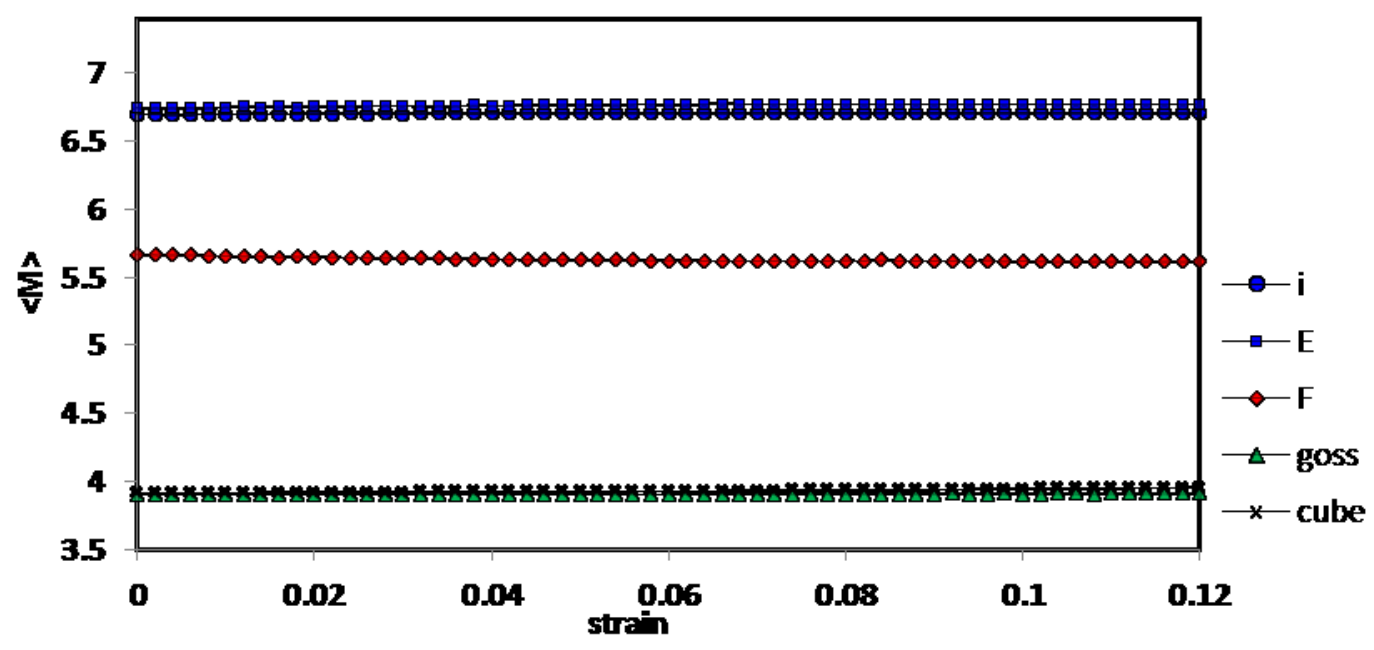

Figure 10. Simulation (VPSC approach) of the mechanical behaviour through tension in RD of individual texture components (BCC).

The simulated curves reveal that the relatively strong rolling texture of the CR sheet (particularly $E$ in gamma fibre and i components) may lead to an increase of the stress through tension in RD. On the contrary, concerning the Hall-Petch relationship [27] [28], a fine microstructure of C-ASR sheets may raise their stress levels higher than $\mathrm{CR}$ sample with a coarse microstructure.

In various total reductions (pre-strain), the factor of fine microstructure of the C-ASR specimen leads to an increase in stress; but in the higher reduction (60\%) where the CR sheet has higher yield strength, the impact of crystallographic texture gets more effective. In other words, due to the small pre-strain of $18 \%$ total thickness reduction, the new textures have not completely developed. While at $60 \%$ total thickness reduction, the fully developed texture affects the mechanical response and increases the stress levels through tension in the rolling direction (Figure 8). 


\section{Conclusions}

Two types of ASR processes were carried out on the interstitial free (IF) steel. This allowed for a study of the impact of shear strain on its macro and micro properties to be conducted. The crystallographic texture evolution as well as microstructure change was taken into consideration. The induced mechanical behaviors of the ASR samples were also evaluated. From the results obtained in this work, the following conclusions can be drawn:

- Texture analyses indicate that the developed texture of CR sample is relatively strong compared to the texture measured from the C-ASR sample. The microstructural analysis using optical microscopy indicates no significant difference in the morphology of two rolled samples by the ASR and CR processes. However, the substructure analyses of the ASR and CR samples observed by TEM and EBSD reveal different features in terms of their morphology and size. The dislocation cells formed during the C-ASR is equiaxed and small compared to those formed during the CR process, and consequently the fine microstructure formed after recovery heat-treatment in C-ASR specimen.

- The effect of strain path change during ASR passes (i.e. R-ASR) on the microstructure was studied: The substructure of the R-ASR sample is not-well-organized compared to that in the C-ASR sample. This can be attributed with the changing of the strain path between the ASR passes. However, the shear plastic deformation through the process led to the formation of (imprecisely) equiaxed dislocation cells in some part of observed region.

- The effect of sheet thickness reduction on the stress-strain curves of the rolled and annealed samples were studied. For low thickness reductions, the stress-strain curve of the C-ASR sample reveals the highest stress. At a $60 \%$ thickness reduction, the highest ultimate strength was recorded for the CR sample; i.e. presenting a transient phenomenon at the $36 \%$ rolling thickness reduction.

\section{Appendix A. $\alpha$ parameter of different processes}

In this part, three different strain paths of C-ASR, R-ASR and CR are investigated. With the purpose of understanding the materials' behavior through strain path change in the second pass of each strain route, the $\alpha$ parameter defined by Schmitt et al. [26] was calculated. $\alpha$ parameter is the cosine of the angle between two vectors representing the first and second strain route and provides an estimate of amplitude of the impact of strain path change on the activity of different slip systems through plastic deformations. This parameter is a unique and non-ambiguous definition for any sequence of two linear strain paths.

$$
\boldsymbol{\alpha}=\frac{\varepsilon_{\text {pre }}: \varepsilon_{\mathbf{p}}}{\left|\varepsilon_{\text {pre }}\right| \cdot\left|\varepsilon_{\mathbf{p}}\right|} \quad \text { Equation } 4
$$


where $\varepsilon_{\text {pre }}$ is the tensor corresponding to the first plastic strain and $\varepsilon_{\mathrm{p}}$ the tensor representing the plastic part of the subsequent deformation.

Here, two passes of CR, as well as two passes of R-ASR and C-ASR are supposed to reduce the sheet thickness. The first and second pass of each process (CR, R-ASR and C-ASR) are designated as $\varepsilon_{\text {pre }}$ and $\varepsilon_{p}$ respectively (Figure 11).

$$
\begin{aligned}
& \text { a) } \mathrm{CR} \\
& {\left[\begin{array}{ccc}
\varepsilon_{11} & 0 & 0 \\
0 & 0 & 0 \\
0 & 0 & -\varepsilon_{11}
\end{array}\right] \quad\left[\begin{array}{ccc}
\varepsilon_{11} & 0 & 0 \\
0 & 0 & 0 \\
0 & 0 & -\varepsilon_{11}
\end{array}\right]} \\
& \text { b) R-ASR } \\
& {\left[\begin{array}{ccc}
\varepsilon_{11} & 0 & \gamma / 2 \\
0 & 0 & 0 \\
\gamma / 2 & 0 & -\varepsilon_{11}
\end{array}\right]} \\
& {\left[\begin{array}{ccc}
\varepsilon_{11} & 0 & -\gamma / 2 \\
0 & 0 & 0 \\
-\gamma / 2 & 0 & -\varepsilon_{11}
\end{array}\right]} \\
& \text { c) C-ASR } \\
& {\left[\begin{array}{ccc}
\varepsilon_{11} & 0 & \gamma / 2 \\
0 & 0 & 0 \\
\gamma / 2 & 0 & -\varepsilon_{11}
\end{array}\right]} \\
& {\left[\begin{array}{ccc}
\varepsilon_{11} & 0 & \gamma / 2 \\
0 & 0 & 0 \\
\gamma / 2 & 0 & -\varepsilon_{11}
\end{array}\right]}
\end{aligned}
$$
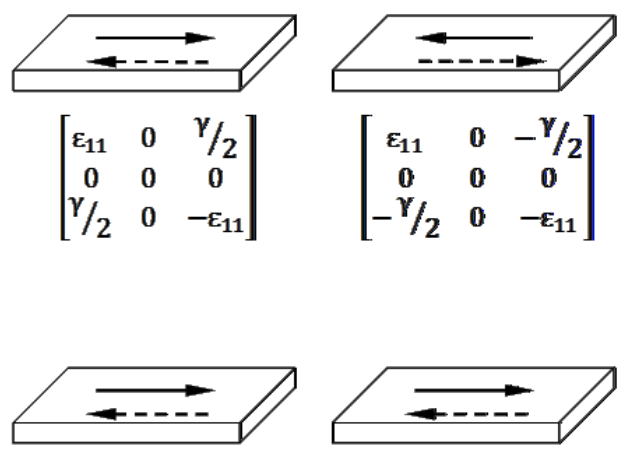

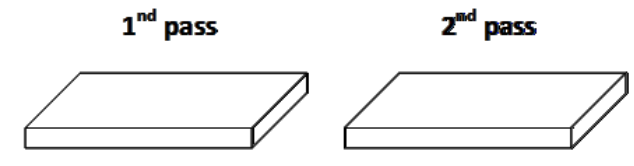

Figure 11. The first and second pass of various strain routes: a) CR, b) R-ASR and c) C-ASR processes.

Here, $\gamma$ is the shear strain imposed through the ASR processes and also $\varepsilon_{11}$ is the compressive strain in deformation routes. According Equation A.1, $\alpha$ parameters for two passes of each process are given as:
a) $\alpha_{C R}=\frac{\varepsilon_{11} \cdot \varepsilon_{11}+\left(-\varepsilon_{11}\right) \cdot\left(-\varepsilon_{11}\right)}{\sqrt{\varepsilon_{11}^{2}+\left(-\varepsilon_{11}\right)^{2}} \cdot \sqrt{\varepsilon_{11}^{2}+\left(-\varepsilon_{11}\right)^{2}}}=1$
b) $\alpha_{R-A S R}=\frac{\varepsilon_{11} \cdot \varepsilon_{11}+\left(-\varepsilon_{11}\right) \cdot\left(-\varepsilon_{11}\right)+(\gamma / 2) \cdot(-\gamma / 2)+(-\gamma / 2) \cdot(\gamma / 2)}{\sqrt{\varepsilon_{11}{ }^{2}+\left(-\varepsilon_{11}\right)^{2}+\left({ }^{\gamma} / 2\right)^{2}+\left(-{ }^{\gamma} / 2\right)^{2}} \cdot \sqrt{\varepsilon_{11}{ }^{2}+\left(-\varepsilon_{11}\right)^{2}+\left(-{ }^{\gamma} / 2\right)^{2}+(\gamma / 2)^{2}}}=\frac{\varepsilon_{11}{ }^{2}-(\gamma / 2)^{2}}{\varepsilon_{11}{ }^{2}+\left({ }^{\gamma} / 2\right)^{2}}<1$
c) $\alpha_{C-A S R}=\frac{\varepsilon_{11} \cdot \varepsilon_{11}+\left(-\varepsilon_{11}\right) \cdot\left(-\varepsilon_{11}\right)+(\gamma / 2) \cdot(\gamma / 2)+(\gamma / 2) \cdot(\gamma / 2)}{\sqrt{\varepsilon_{11}{ }^{2}+\left(-\varepsilon_{11}\right)^{2}+\left(\gamma^{\gamma} / 2\right)^{2}+\left(\gamma^{\gamma} / 2\right)^{2}} \cdot \sqrt{\varepsilon_{11}{ }^{2}+\left(-\varepsilon_{11}\right)^{2}+(\gamma / 2)^{2}+\left({ }^{\gamma} / 2\right)^{2}}}=\frac{\varepsilon_{11}{ }^{2}+(\gamma / 2)^{2}}{\varepsilon_{11}{ }^{2}+(\gamma / 2)^{2}}=1$ Equation 5 
The calculated $\alpha$ parameters for two passes of CR and C-ASR equal 1, which means the strain path did not change between the passes and dislocations glide on the same slip systems used in the previous pass. In the R-ASR process, however, the $\alpha$ parameter is less than 1 which depends on the magnitude of $\gamma$ relative to $\varepsilon_{11}$; e.g. the $\alpha$ parameter of two passes of R-ASR process equals zero for the value $\varepsilon_{11}=\frac{\gamma}{2}$. For $\alpha$ parameters close to zero, the new slip systems which were latent in the first pass become active.

It should be noted that in the calculations, it was assumed that the ratio of $\frac{\varepsilon_{11}}{\gamma}$ is the same for both passes of

ASR processes. The change of $\frac{\varepsilon_{11}}{\gamma}$ ratio may also lead to having a different strain path in the second pass of C-ASR.

\section{Acknowledgment}

The author would like to acknowledge Portuguese Foundation of Science and Technology (FCT) for grant with reference SFRH/45835/2008.

\section{References}

[1] H. S. Kim, M. H. Seo and S. I. Hong, "Plastic deformation analysis of metals during equal channel angular pressing," Journal of Materials Processing Technology, vol. 113(1), pp. 622-626., 2001.

[2] Y. Saito, N. Tsuji, H. Utsunomiya, T. Sakai and R. G. Hong, "Ultra-fine grained bulk aluminum produced by accumulative roll-bonding (ARB) process," Scripta Mater, vol. 39(9), pp. 1221-1227., 1998.

[3] Z. Horita, D. J. Smith, M. Furukawa, M. Nemoto, R. Z. Valiev and T. G. Langdon, "An investigation of grain boundaries in submicrometer-grained $\mathrm{Al}-\mathrm{Mg}$ solid solution alloys using high-resolution electron microscopy.," Journal of Materials Research, vol. 11(08): , pp. 1880-1890, 1996.

[4] J. K. Lee and D. N. Lee, "Shear texture development and grain refinement in asymmetrically rolled aluminum alloy sheets by varied reduction per pass," Materials Science Forum, vol. 408 , no. Trans Tech Publications., pp. 1419-1424, 2002. 
[5] K. H. Kim and D. N. Lee, "Analysis of deformation textures of asymmetrically rolled aluminum sheets," Acta Materialia, vol. 49(13), pp. 583-2595, 2001.

[6] H. Utsunomiya, T. Ueno and T. Sakai, "Improvement in the r-value of aluminum sheets by differential-friction rolling," Scripta Materialia, vol. 57(12), pp. 1109-1112, 2007.

[7] F. J. Simões, R. J. A. de Sousa, J. J. Grácio, F. Barlat and J. W. Yoon, "Mechanical behavior of an asymmetrically rolled and annealed 1050-0 sheet," International journal of mechanical sciences, vol. 50(9), pp. 1372-1380, 2008.

[8] S. Tamimi, J. P. Correia, A. B. Lopes, S. Ahzi, F. Barlat and J. J. Gracio, "Asymmetric rolling of thin AA-5182 sheets: Modelling and experiments.," Materials Science and Engineering: A, vol. 603, pp. 150-159, 2014.

[9] S. H. Lee and D. N. Lee, "Analysis of deformation textures of asymmetrically rolled steel sheets.," International Journal of Mechanical Sciences, vol. 43(9), pp. 1997-2015, 2001.

[10] A. Wauthier, H. Regle, J. Formigoni and G. Herman, "The effects of asymmetrical cold rolling on kinetics, grain size and texture in IF steels.," Materials Characterization, vol. 60(2), pp. 90-95, 2009.

[11] Y. Ding, J. Jiang and A. Shan, "Microstructures and mechanical properties of commercial purity iron processed by asymmetric rolling.," Materials Science and Engineering: A, vol. 509(1), pp. 7680, 2009.

[12] M. H. Cai, S. S. Dhinwal, Q. H. Han, Q. Chao and P. D. Hodgson, "radient ultrafine ferrite and martensite structure and its tensile properties by asymmetric rolling in low carbon microalloyed steel.," Materials Science and Engineering: A, , vol. 583, pp. 205-209, 2013.

[13] A. Molinari, G. R. Canova and S. Ahzi, "Molinari, A., Canova, G.R. and Ahzi, S., 1987. A self consistent approach of the large deformation polycrystal viscoplasticity.," Acta Metallurgica, vol. 35(12), pp. 2983-2994, 1987. 
[14] R. Lebensohn and C. N. Tomé, "A self-consistent viscoplastic model: prediction of rolling textures of anisotropic polycrystals.," Materials Science and Engineering: A, Vols. 175(1-2), pp. 71-82, 1994.

[15] C. Tome, G. R. Canova, U. F. Kocks, N. Christodoulou and J. J. Jonas, "he relation between macroscopic and microscopic strain hardening in FCC polycrystals.," Acta metallurgica,, vol. 32(10), pp. 1637-1653., 1984.

[16] H. J. Bunge, Texture analysis in materials science: mathematical methods., Elsevier, 2013.

[17] M. Hölscher, D. Raabe and K. Lücke, "Rolling and recrystallization textures of bcc steels.," Steel Research International, vol. 62(12), pp. 567-575, 1991.

[18] Y. T. Zhu and T. C. Lowe, "Observations and issues on mechanisms of grain refinement during ECAP process.," Materials Science and Engineering: A, vol. 291(1), pp. 46-53, 2000.

[19] D. H. Shin, I. Kim, J. Kim and K. T. Park, "Grain refinement mechanism during equal-channel angular pressing of a low-carbon steel.," Acta Materialia, vol. 9(7), pp. 1285-1292, 2001.

[20] Y. Saito, H. Utsunomiya, N. Tsuji and T. Sakai, “Novel ultra-high straining process for bulk materials development of the accumulative roll-bonding (ARB) process.," Acta materialia, vol. 47(2), pp. 579-583, 1999.

[21] S. Tamimi, M. Ketabchi and N. Parvin, "Microstructural evolution and mechanical properties of accumulative roll bonded interstitial free steel.," Materials \& Design, vol. 30(7), pp. 2556-2562, 2009.

[22] Q. Cui and K. Ohori, "Grain refinement of high purity aluminium by asymmetric rolling.,," Materials Science and Technology, vol. 16(10), pp. 1095-1101, 2000.

[23] Y. Iwahashi, Z. Horita, M. Nemoto and T. G. Langdon, "An investigation of microstructural evolution during equal-channel angular pressing.," Acta materialia, vol. 45(11), pp. 4733-4741, 1997. 
[24] Y. Iwahashi, Z. Horita, M. Nemoto and T. G. Langdon, "The process of grain refinement in equalchannel angular pressing.," Acta materialia, vol. 46(9), pp. 3317-3331, 1998.

[25] A. Gholinia, P. B. Prangnell and M. V. Markushev, "The effect of strain path on the development of deformation structures in severely deformed aluminium alloys processed by ECAE.," Acta Materialia, vol. 48(5), pp. 1115-1130, 2000.

[26] J. H. Schmitt, E. L. Shen and J. L. Raphanel, "A parameter for measuring the magnitude of a change of strain path: validation and comparison with experiments on low carbon steel.," International Journal of Plasticity, vol. 10(5), pp. 535-551, 1994.

[27] E. O. Hall, "The deformation and ageing of mild steel: III discussion of results.," Proceedings of the Physical Society-Section B, vol. 64(9), p. 747, 1951.

[28] N. J. Petch, "The Cleavage Strengh of Polycrystals.," J. of the Iron and Steel Inst, vol. 174, pp. 25$28,1953$. 